\title{
Investigation of Spectrum and Analysis of Risk Factor for Gastrointestinal Diseases of Chinese Army During a Field Training on the Midland of China in the New Era
}

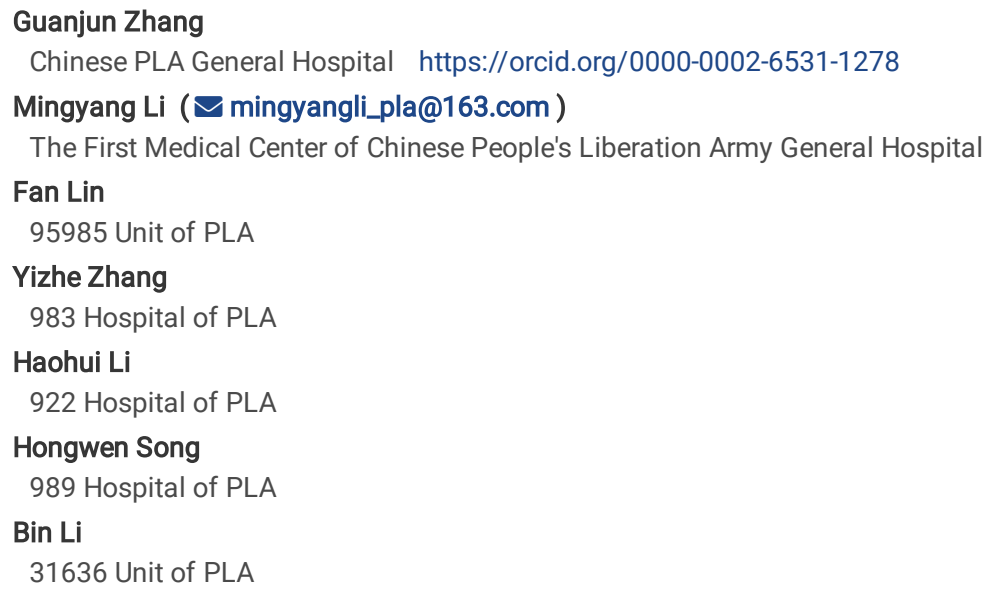




\section{Abstract}

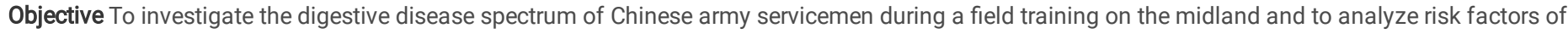
diseases in order to discuss the relative prevention and treatment strategies.

Methods Seven troops during a field training on the midland of China were randomly selected by using stratified cluster random sampling methods,all soldiers in that received a questionnaire survey,and then we collected all questionnaires and summarized the soldiers' digestive system disease spectrum, and discussed risk factors for diseases.

Results 3055 valid questionnaires were finally collected, 896 soldiers had digestive symptoms, and of all symptoms diarrhea was the most common,whose ratio was 29.3\%; 507 soldiers suffered from digestive system diseases, including gastritis,enteritis,digestive ulcer,functional bowel disease, which were more common.By the logistic regression analysis, length of military service time,identity of army officer,length of a field training time,training pressure,chilled abdomen,and defiled food were possible risk factors for digestive diseases.

Conclusions During a field training,a healthy and regular diet, the thermal outfit,appropriate decompression of negative emotions and reasonable training time may effectively decrease the risk of digestive diseases, so that to reduce attrition.

\section{Introduction}

Diseases of the digestive system have a high incidence among internal medical diseases. According to the research of depletion of Chinese soldier numbers in modern wars, digestive system diseases also contributed to a high incidence in wartime. In Chinese Resistance War against Japanese Aggression and China National War of Liberation, the incidence of digestive diseases took the first place among all internal medical diseases, and it took the second place in the war to resist U.S. aggression and aid Korea and Sino-Vitnamese War ${ }^{[1]}$.In the war to resist U.S. aggression and aid Korea, the ratio of Chinese soldiers hospitalized for digestive diseases to internal diseases was $30.2 \%$, which was significantly higher than $19.3 \%$ of China domestic troops ${ }^{[1]}$. At that time, due to the poor training conditions and poor medical cares, these four types of digestive diseases,gastroenteritis, stress ulcer, functional abdominal pain and gastrointestinal bleeding, were the most common ${ }^{[1-2]}$. Since the 19th National Congress of the Communist Party of China, China has entered a new era, and the reform of armed forces has made steady progress.Army soldiers are more highly trained and submitted to more strictly imposed discipline than before, but the life conditions and medical cares are significantly better. However,there are few studies on the investigation and analysis of the digestive diseases spectrum change of Chinese soldiers in recent years. So the aim of our study is to investigate the new digestive system disease spectrum of army soldiers during a field training in the midland of China, and then to look for relevant strategies for disease control and prevention, which may reduce non-combat attrition and improve the forces fighting capacity.

\section{Materials And Methods}

\section{Objects}

According to the results of previous research, the incidence of digestive diseases in the Chinese general population is about $30 \%$. By using this as a reference baseline prevalence rate, with an accuracy of $\pm 2 \%$, the sample size of this study is estimated to be at least 2,017 . Finally, a total of 3,055 army servicemen during a field training in the midland of China from January 2021 to May 2021 were selected as investigation objects in our study.So our study meets the requirement of sample size.

\section{Methods}

One basic unit was selected from each of seven provinces in the midland of China by the stratified cluster random sampling method. Questionnaire survey was carried out on all military officers and soldiers in the units by military doctors who had a good command of medical knowledge and received professional training before the survey. The datas from the questionnaires were recorded and input into the excel by one person and meanwhile were checked by another one to ensure the authenticity and validity of results.

\section{Questionnaire}

The questionnaire was designed by gastroenterologists, epidemiologists and statistics experts on the foundation of literature review. And the contents of this questionnaire include five aspects: (1)Basic information: Gender, age, height, weight, educational background, military services, military service time, status, smoking, diet habits, family history of digestive diseases and use history of NSAIDs(aspirin, clopidogrel, ibuprofen,etc.); (2)Training and education: the daily military training time, education on digestive disease prevention knowledge; (3)digestive diseases: acute gastroenteritis, digestive ulcer, gastrointestinal bleeding, inflammatory bowel disease, functional bowel disease, gastroesophageal reflux disease, pancreatitis, abnormal liver function nausea;(4)Related symptoms: vomiting, abdominal distension, diarrhea, abdominal pain, constipation, poor appetite, acid reflux;(5)Treatment: medical institutions, examination items, treatment effect, etc.

Before a formal investigation, we had to check the reliability and validity of the questionnaire. So the questionnaires were distributed to 100 people and then 98 valid questionnaires were collected. The datas were input into IBM SPSS Statistics Software. Through reliability analysis the questionnaire, Crobach's a coefficient was calculated to be 0.86 , so the reliability of the questionnaire was good. Through the validity analysis of the questionnaire, the results that KMO 
value $₫ 0.6$, the significance of Bartlett test of spherical degree $₫ 0.01$ and load coefficient of each item $\varangle 0.05$ were obtained, so the validity of the questionnaire was also good.

\section{Statistical analysis}

The valid questionnaire information was input into the excel form to establish a database. And descriptive statistical analysis was carried out by SPSS 26 software. Counting datas were tested by $X^{2}$ test. Grade datas were tested by Mann-Whitney test. The correlation analysis on risk factors of the digestive diseases was conducted by logistics regression analysis. If $\mathrm{P} \otimes 0.05$, the difference was statistically significant.

\section{Results}

\section{The distributed questionnaires}

A total of 3,200 questionnaires were sent out,and then 3,070 were collected, and finally 3,055 were valid through the check, so an effective rate of this survey was $95.5 \%$.

\section{Servicemen's Symptom and disease spectrum of digestive system in the field training}

A total of 896 patients had digestive symptoms, with a prevalence of $29.3 \%$, and diarrhea was the most common symptom, with a prevalence of $13.7 \%$. A total of 507 patients ever had digestive diseases during a field training period in the midland, with a prevalence of $16.6 \%$, among which, gastritis, enteritis, gastrointestinal ulcers and functional bowel diseases were more common. The details are listed in Table 1 and 2.

Table 1

Symptom spectrum of digestive system

\begin{tabular}{|llllll|}
\hline Symptom & No.(of all population) & $\%$ & Symptom & No.(of all population) & $\%$ \\
\hline Vomit & 199 & 6.5 & Poor appetite & 268 & 8.8 \\
\hline Abdominal pain & 208 & 6.8 & Acid reflux & 169 & 5.5 \\
\hline Abdominal distension & 229 & 7.5 & constipation & 289 & 9.5 \\
\hline diarrhea & 419 & 13.7 & asymptomatic & 2159 & 70.7 \\
\hline
\end{tabular}

Table 2

Disease spectrum of digestive system

\begin{tabular}{|llllll|}
\hline Disease & No.(of all population) & $\%$ & Disease & No.(of all population) & $\%$ \\
\hline Acute or chronic gastritis & 317 & 10.4 & Irritable bowel syndrome & 40 & 1.3 \\
\hline Acute or chronic enteritis & 200 & 6.5 & Functional bowel disease & 90 & 2.9 \\
\hline gastrointestinal ulcers & 110 & 3.6 & Abnormal hepatic function & 10 & 0.3 \\
\hline Gastrointestinal bleeding & 20 & 0.7 & Acute pancreatitis & 10 & 0.3 \\
\hline Inflammatory bowel disease & 10 & 0.3 & No diseases & 2548 & 83.4 \\
\hline Gastroesophageal reflux disease & 10 & 0.3 & & & \\
\hline
\end{tabular}

\section{Comparison of symptoms and diseases of digestive system among different groups}

There were significant differences in the incidence of digestive symptoms and diseases of servicemen among different groups of age, military service time, status, smoking amount,digestive disease family history,the field training time, irregular diet times,training pressure,chilled abdomen,defiled food history and disease prevention knowledge education(Pष0.01). There was no significant difference in the incidence of digestive symptoms and diseases between different groups of NSAIDs use history(P凶0.05). The details are listed in Table 3 and 4.

Table 3

Comparison of symptoms among different groups $\llbracket$ No./\%区 


\begin{tabular}{|c|c|c|c|c|c|c|c|c|c|c|}
\hline Group & Vomit & $\begin{array}{l}\text { Abdominal } \\
\text { pain }\end{array}$ & $\begin{array}{l}\text { Abdominal } \\
\text { distention }\end{array}$ & Diarrhea & $\begin{array}{l}\text { Poor } \\
\text { appetite }\end{array}$ & $\begin{array}{l}\text { Acid } \\
\text { reflux }\end{array}$ & constipation & asymptomatic & $x^{2}$ & $\mathrm{P}$ \\
\hline \multicolumn{11}{|l|}{ Age } \\
\hline $18-30$ & 169ه6.5》 & 178(6.9) & $129(5.0)$ & 299(11.6) & 188(7.3) & $119(4.6)$ & $209(8.1)$ & 1920(74.2) & 115.3 & 0.00 \\
\hline $31-40$ & $20(4.9)$ & $10(2.4)$ & $80(19.5)$ & $80(19.5)$ & 70(17.1) & $40(9.8)$ & $60(14.9)$ & $220(53.7)$ & & \\
\hline $41-50$ & 10(16.9) & 20(33.9) & 20(33.9) & $40(67.8)$ & 10(16.9) & 10() 16.9 & 20(33.9) & 19(32.2) & & \\
\hline \multicolumn{11}{|c|}{ Military service time } \\
\hline 2 years & $30(3.9)$ & $29(3.8)$ & $10(1.3)$ & $20(2.6)$ & $40 \rrbracket 5.2 \rrbracket$ & 0 & 29®3.8\ & $684 \llbracket 88.5 \rrbracket$ & 216.4 & 0.00 \\
\hline $2-5$ years & $79(9.9)$ & $60(7.5)$ & $49(6.2)$ & 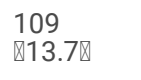 & $78 \llbracket 9.8 \rrbracket$ & $69 \llbracket 8.7 \rrbracket$ & $40 \bigotimes 5.0 \rrbracket$ & $557 \bigotimes 70.1 \rrbracket$ & & \\
\hline $6-10$ years & $50(5.6)$ & $59(6.6)$ & $60(6.7)$ & 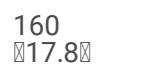 & 60ه6.7ه & $60 \rrbracket 6.7 \rrbracket$ & 120ه13.3ه & $610 \otimes 67.9 \rrbracket$ & & \\
\hline $\begin{array}{l}\text { More than } 10 \\
\text { years }\end{array}$ & $40(6.8)$ & $60(10.2)$ & $110(18.7)$ & 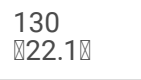 & $90 \rrbracket 15.3 \rrbracket$ & $40 \rrbracket 6.8 \rrbracket$ & 100ه17.0区 & $308 \llbracket 52.4 \rrbracket$ & & \\
\hline \multicolumn{11}{|l|}{ Status } \\
\hline $\begin{array}{l}\text { Commanding } \\
\text { officer }\end{array}$ & $30 \otimes 13.0 \bigotimes$ & $10 \otimes 4.3 \rrbracket$ & 20ه8.7凶 & $80 \rrbracket 34.8 \rrbracket$ & $20 \rrbracket 8.7 \rrbracket$ & $20 \rrbracket 8.7 \rrbracket$ & $20 \rrbracket 8.7 \rrbracket$ & $110 \otimes 47.8 \rrbracket$ & 303.5 & 0.00 \\
\hline $\begin{array}{l}\text { Technical } \\
\text { officers }\end{array}$ & $20 \otimes 8.0 \rrbracket$ & $50 \rrbracket 20.1 \rrbracket$ & 40囚16.1】 & $80 \rrbracket 32.1 \rrbracket$ & $20 \rrbracket 8.0 \rrbracket$ & $10 \rrbracket 4.0 \rrbracket$ & $20 \rrbracket 8.0 \rrbracket$ & $129 \llbracket 51.8 \rrbracket$ & & \\
\hline Sergeant & 139ه7.8ه & $119 \otimes 6.7 \rrbracket$ & $169 \otimes 9.5 \rrbracket$ & $\begin{array}{l}249 \\
\square 14.00\end{array}$ & $198 \otimes 11.2 \rrbracket$ & $\begin{array}{l}139 \\
87.8 \square\end{array}$ & $230 \otimes 13.08$ & $1177 \otimes 66.3 \rrbracket$ & & \\
\hline Conscript & $10 \otimes 1.2 \rrbracket$ & $29 \llbracket 3.6 \rrbracket$ & 0 & $10 \otimes 1.2 \rrbracket$ & $30 \llbracket 3.7 \rrbracket$ & 0 & $19 \llbracket 2.4 \rrbracket$ & $743 \llbracket 92.6 \rrbracket$ & & \\
\hline \multicolumn{11}{|l|}{ Daily smoking } \\
\hline $\begin{array}{l}\text { More than } 20 \\
\text { cigarettes }\end{array}$ & 0 & $30 \rrbracket 30.0 \rrbracket$ & 10ه10.0ه & $10 \otimes 10.0 \rrbracket$ & $20 \otimes 20.0 \rrbracket$ & 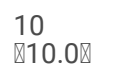 & 10冈10.0ष & 70ه70.0ه & 37.3 & 0.00 \\
\hline $10-20$ cigarettes & $20 \otimes 4.9 \rrbracket$ & $20 \rrbracket 4.9 \rrbracket$ & $40 \rrbracket 9.8 \rrbracket$ & $60 \rrbracket 14.7 \rrbracket$ & $39 \llbracket 9.6 \rrbracket$ & $10 \rrbracket 2.5 \rrbracket$ & $40 \rrbracket 9.8 \rrbracket$ & $269 \llbracket 65.9 \rrbracket$ & & \\
\hline $1-10$ cigarettes & $30 \rrbracket 3.3 \rrbracket$ & $29 \llbracket 3.2 \rrbracket$ & $30 \rrbracket 3.3 \rrbracket$ & $70 \rrbracket 7.6 \rrbracket$ & $60 \rrbracket 6.6 \rrbracket$ & $40 \rrbracket 4.4 \rrbracket$ & 80冈8.7ه & 717ه78.3囚 & & \\
\hline No cigarette & $30 \rrbracket 3.3 \rrbracket$ & $129 ه 7.98$ & $149 \otimes 9.1 \rrbracket$ & $\begin{array}{l}279 \\
\square 17.1 \rrbracket\end{array}$ & 149ه9.1ه & 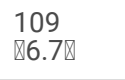 & 159ه9.7区 & 1103囚67.6ه & & \\
\hline \multicolumn{11}{|l|}{ Family history } \\
\hline With & $29 \otimes 9.7 \rrbracket$ & $60 \rrbracket 20.1 \rrbracket$ & $39 ه 13.0 \rrbracket$ & $49816.4 \rrbracket$ & 59ه19.7ه & 29ष9.7凹 & $40 \rrbracket 13.4 \rrbracket$ & $140 \rrbracket 46.8 \rrbracket$ & 90.8 & 0.00 \\
\hline Without & $170 \rrbracket 6.2 \rrbracket$ & $148 \bigotimes 5.4 \rrbracket$ & $190 \otimes 6.9 \rrbracket$ & $\begin{array}{l}370 \\
\square 13.4 \square\end{array}$ & $209 \otimes 7.6 \rrbracket$ & 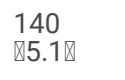 & $249 \varangle 9.0 \otimes$ & 2019ه73.3区 & & \\
\hline \multicolumn{11}{|c|}{ NSAIDs use history } \\
\hline with & $10 \otimes 12.5 \rrbracket$ & 0 & 0 & 0 & 0 & 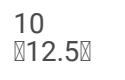 & 0 & $60 \otimes 75.0 \rrbracket$ & 0.743 & 0.389 \\
\hline without & 189ه6.4】 & $208 \otimes 7.0 \rrbracket$ & $229 ه 7.7 \rrbracket$ & 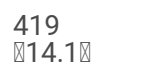 & $268 \otimes 9.0 \bigotimes$ & 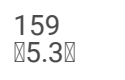 & 289ه9.7】 & 2099ه70.6囚 & & \\
\hline \multicolumn{11}{|l|}{ Field training time } \\
\hline Within 15 days & $40 \rrbracket 4.0 \rrbracket$ & 19ه1.9凶 & $50 \rrbracket 5.0 \rrbracket$ & $70 \rrbracket 6.9 ه$ & 80冈7.9凶 & $40 \rrbracket 4.0 \bigotimes$ & $6986.8 \rrbracket$ & $809 \llbracket 80.3 \rrbracket$ & 270.3 & 0.00 \\
\hline $16-30$ days & $10 \otimes 4.0 \rrbracket$ & 40囚16.0区 & $50 \rrbracket 20.0 \rrbracket$ & $30 \otimes 12.0 \rrbracket$ & $30 \rrbracket 12.0 \rrbracket$ & $\begin{array}{l}30 \\
\square 12.0 \bigotimes\end{array}$ & $60 \rrbracket 24.0 \rrbracket$ & $90 \rrbracket 36.0 \rrbracket$ & & \\
\hline $1-3$ months & $40 \otimes 7.2 \rrbracket$ & 49₫8.8\ & $20 \rrbracket 3.6 \rrbracket$ & $\begin{array}{l}120 \\
\otimes 21.6 \rrbracket\end{array}$ & $30 \otimes 5.4 \rrbracket$ & $10 \bigotimes 1.8 \rrbracket$ & $40 \otimes 7.2 \rrbracket$ & $367 \rrbracket 66.0 \rrbracket$ & & \\
\hline 4-6 months & $39 \llbracket 4.2 \rrbracket$ & 30囚3.3囚 & $79 \otimes 8.6 \rrbracket$ & 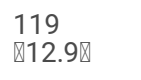 & $98 \bigotimes 10.6 \rrbracket$ & 49₫5.3囚 & 70ه7.6区 & $723 \bigotimes 78.5 \rrbracket$ & & \\
\hline $\begin{array}{l}\text { More than } 6 \\
\text { months }\end{array}$ & $70 \otimes 21.9 \rrbracket$ & 70ه21.9凶 & $30 \otimes 9.4 \rrbracket$ & $80 \rrbracket 25.0 \rrbracket$ & $30 \rrbracket 9.4 \rrbracket$ & $\begin{array}{l}40 \\
\square 12.5 \rrbracket\end{array}$ & $50 \rrbracket 15.6 \rrbracket$ & $170 \rrbracket 53.1 \rrbracket$ & & \\
\hline \multicolumn{11}{|l|}{ Irregular diet time } \\
\hline 4 days per week & $60 \otimes 43.5 \rrbracket$ & $9 \llbracket 6.5 \rrbracket$ & $9 \llbracket 6.5 \rrbracket$ & $29 \otimes 21.0 \rrbracket$ & $28 \varangle 21.0 \rrbracket$ & 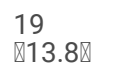 & $29 \varangle 21.0 \rrbracket$ & $59 \varangle 43.5 \rrbracket$ & 334.8 & 0.00 \\
\hline $\begin{array}{l}2-3 \text { days per } \\
\text { week }\end{array}$ & $\begin{array}{l}208 \\
\square 41.0 \bigotimes\end{array}$ & 100®19.7凶 & 100®19.7凶 & 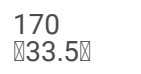 & 99囚19.5区 & $50 \rrbracket 9.9 \rrbracket$ & $90 \otimes 17.8 \rrbracket$ & $208 \bowtie 41.0 \rrbracket$ & & \\
\hline
\end{tabular}




\begin{tabular}{|c|c|c|c|c|c|c|c|c|c|c|}
\hline Without & 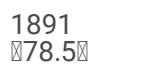 & $99 \llbracket 4.1 \rrbracket$ & $120 \otimes 5.0 \bigotimes$ & $220 \otimes 9.1 \rrbracket$ & $139 \llbracket 5.9 \rrbracket$ & 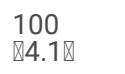 & 170ه7.1区 & $1890 \otimes 78.5 \rrbracket$ & & \\
\hline \multicolumn{11}{|c|}{ High training pressure } \\
\hline 4 days per week & $40 \bigotimes 22.2 \rrbracket$ & $50 \rrbracket 27.8 \rrbracket$ & $40 \rrbracket 22.2 \rrbracket$ & $60 \rrbracket 33.3 \rrbracket$ & $30 \otimes 16.7 \rrbracket$ & $10 \otimes 5.6 \bigotimes$ & $20 \rrbracket 11.1 \rrbracket$ & $80 \rrbracket 44.4 \rrbracket$ & 266.7 & 0.00 \\
\hline $\begin{array}{l}2-3 \text { days per } \\
\text { week }\end{array}$ & $50 \otimes 12.0 \bigotimes$ & $58 \rrbracket 13.9 \rrbracket$ & $50 \otimes 12.0 \rrbracket$ & $70 \otimes 16.8 \rrbracket$ & $60 \bigotimes 14.4 \rrbracket$ & $\begin{array}{l}60 \\
\square 14.4 \rrbracket\end{array}$ & $79(18.9)$ & $179(42.9)$ & & \\
\hline Without & $109(4.4)$ & $100(4.1)$ & 139(5.7) & $289(11.8)$ & $178(7.2)$ & $99(4.0)$ & $190(7.7)$ & $1900(77.3)$ & & \\
\hline \multicolumn{11}{|c|}{ Time when a serviceman has a chilled abdomen } \\
\hline 4 days per week & $30(25.0)$ & $40(33.3)$ & $20(16.7)$ & $60(50.0)$ & 0 & $10(8.3)$ & $20(16.7)$ & $40(33.3)$ & 221.8 & 0.00 \\
\hline $\begin{array}{l}2-3 \text { days per } \\
\text { week }\end{array}$ & $70(22.1)$ & $68(21.5)$ & $60(18.9)$ & $70(22.1)$ & $100(31.5)$ & $40(12.6)$ & $69(21.8)$ & 139(43.8) & & \\
\hline Without & $99(3.8)$ & $100(3.8)$ & 149(5.7) & $289(11.0)$ & $168(6.4)$ & $119(4.5)$ & $200(7.6)$ & $1980(75.6)$ & & \\
\hline \multicolumn{11}{|c|}{ Defiled food history } \\
\hline With & $110(17.2)$ & $120(18.8)$ & $110(17.2)$ & $210(32.9)$ & $139(21.8)$ & $70(11.0)$ & $90(14.1)$ & $230(36.0)$ & 468.7 & 0.00 \\
\hline Without & 89(3.7) & $88(3.6)$ & $119(4.9)$ & $209(8.7)$ & $129(5.3)$ & $99(4.1)$ & 199(8.2) & $1929(79.8)$ & & \\
\hline \multicolumn{11}{|c|}{ Prevention knowledge education } \\
\hline With & $109(5.4)$ & $108(5.3)$ & $109(5.4)$ & $299(14.9)$ & $138(6.9)$ & $119(5.9)$ & $130(6.5)$ & 1474(73.7) & 25.1 & 0.00 \\
\hline Without & $90(8.5)$ & $100(9.4)$ & $119(11.3)$ & $120(11.4)$ & $130(12.3)$ & $50(4.7)$ & $159(15.1)$ & $685(65.0)$ & & \\
\hline
\end{tabular}

Table 4

Comparison of disease spectrum among different groups $₫$ No./\%】 


\begin{tabular}{|c|c|c|c|c|c|c|c|c|c|}
\hline Group & $\begin{array}{l}\text { Acute or } \\
\text { chronic } \\
\text { gastritis }\end{array}$ & $\begin{array}{l}\text { Acute or } \\
\text { chronic } \\
\text { enteritis }\end{array}$ & $\begin{array}{l}\text { Gastrointestinal } \\
\text { ulcer }\end{array}$ & $\begin{array}{l}\text { Gastrointestinal } \\
\text { bleeding }\end{array}$ & $\begin{array}{l}\text { Inflammatory } \\
\text { bowel } \\
\text { disease }\end{array}$ & $\begin{array}{l}\text { Gastroesophageal } \\
\text { reflux disease }\end{array}$ & $\begin{array}{l}\text { Irritable } \\
\text { bowel } \\
\text { syndrome }\end{array}$ & $\begin{array}{l}\text { Functional } \\
\text { bowel } \\
\text { disease }\end{array}$ & $\begin{array}{l}\text { Abnorma } \\
\text { hepatic } \\
\text { function }\end{array}$ \\
\hline \multicolumn{10}{|l|}{ Age } \\
\hline $18-30$ & $257 \otimes 9.9 \rrbracket$ & 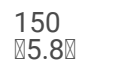 & $80 \rrbracket 3.1 \rrbracket$ & $10 \otimes 0.4 \rrbracket$ & 0 & $10 \otimes 0.4 \rrbracket$ & $20 \rrbracket 0.8 \rrbracket$ & $40 \otimes 1.5 \bigotimes$ & 0 \\
\hline $31-40$ & 30ه7.3ه & $30 \otimes 7.3 \rrbracket$ & $20 \rrbracket 4.9 \rrbracket$ & $10(2.4)$ & 0 & 0 & 0 & $30(7.3)$ & $10(2.4)$ \\
\hline $41-50$ & $30(50.8)$ & 20(33.9) & 10(16.9) & 0 & 10(16.9) & 0 & $20(33.9)$ & 20(33.9) & 0 \\
\hline \multicolumn{10}{|c|}{ Military service time } \\
\hline 2 years & $9(1.2)$ & 0 & 0 & 0 & 0 & 0 & 0 & $1(0.1)$ & 0 \\
\hline $2-5$ years & $70(8.8)$ & $80(10.1)$ & $40(5.0)$ & $10(1.3)$ & 0 & $10(1.3)$ & 10(1.3) & $10(1.3)$ & 0 \\
\hline $6-10$ years & 139(15.5) & $60(6.7)$ & $40(4.4)$ & 0 & 0 & 0 & $10(1.1)$ & $10(1.1)$ & 0 \\
\hline $\begin{array}{l}\text { More than } 10 \\
\text { years }\end{array}$ & $99(16.8)$ & $60(10.2)$ & $30(5.1)$ & $10(1.7)$ & $10(1.7)$ & 0 & $20(3.4)$ & $70(11.9)$ & $10(1.7)$ \\
\hline \multicolumn{10}{|l|}{ Status } \\
\hline $\begin{array}{l}\text { Commanding } \\
\text { officer }\end{array}$ & $50(21.7)$ & $30(13.0)$ & $10(4.3)$ & 0 & 0 & 0 & $1(0.1)$ & $10(4.3)$ & $1(0.1)$ \\
\hline $\begin{array}{l}\text { Technical } \\
\text { officers }\end{array}$ & $50(20.1)$ & $30(12.0)$ & $20(8.0)$ & 0 & $10(4.0)$ & 0 & $40(16.1)$ & $30(12.0)$ & 0 \\
\hline Sergeant & 198(11.2) & $130(7.3)$ & $70(3.9)$ & $20(1.1)$ & 0 & $10(0.6)$ & 0 & $50(2.8)$ & $9(0.6)$ \\
\hline Conscript & 19(2.4) & $10(1.2)$ & $10(1.2)$ & 0 & 0 & 0 & 0 & 0 & 0 \\
\hline \multicolumn{10}{|l|}{ Daily smoking } \\
\hline $\begin{array}{l}\text { More than } 20 \\
\text { cigarettes }\end{array}$ & $20(20.0)$ & $10(10.0)$ & $20(20.0)$ & 0 & 0 & 0 & 0 & $10(10.0)$ & 0 \\
\hline $\begin{array}{l}10-20 \\
\text { cigarettes }\end{array}$ & $49(12.0)$ & $30(7.4)$ & $10(2.5)$ & 0 & 0 & 0 & 0 & $10(10.0)$ & 0 \\
\hline $\begin{array}{l}1-10 \\
\text { cigarettes }\end{array}$ & $49(5.3)$ & $50(5.5)$ & $20(2.2)$ & 0 & 0 & 0 & 0 & $10(1.1)$ & $1(0.1)$ \\
\hline No cigarette & 199(12.2) & $110(6.7)$ & $60(3.7)$ & $20(1.2)$ & $10(0.6)$ & $10(0.6)$ & $40(2.5)$ & $60(3.7)$ & $9(0.6)$ \\
\hline \multicolumn{10}{|l|}{ Family history } \\
\hline With & $30(10.0)$ & $30(10.0)$ & $40(13.4)$ & 0 & 0 & 0 & 10(3.3) & $10(3.3)$ & 0 \\
\hline Without & $287(10.4)$ & $170(6.2)$ & $70(2.5)$ & $20(0.7)$ & $10(0.4)$ & $10(0.4)$ & $30(1.1)$ & $80(2.9)$ & $10(0.4)$ \\
\hline \multicolumn{10}{|c|}{ NSAIDs use history } \\
\hline with & $2(0.1)$ & 0 & $5(0.2)$ & $3(0.1)$ & 0 & 0 & 0 & 0 & 0 \\
\hline without & $315(10.6)$ & $200(6.7)$ & 105(3.6) & $17(0.6)$ & $10(0.3)$ & $10(0.3)$ & $40(1.3)$ & $90(3.0)$ & $10(0.3)$ \\
\hline \multicolumn{10}{|c|}{ Field training time } \\
\hline $\begin{array}{l}\text { Within } 15 \\
\text { days }\end{array}$ & $49(4.9)$ & $30(3.0)$ & $10(1.0)$ & 0 & $10(1.0)$ & 0 & $30(3.0)$ & $20(2.0)$ & 0 \\
\hline $16-30$ days & $40(16.0)$ & 0 & $10(4.0)$ & 0 & 0 & 0 & $10(4.0)$ & $20(8.0)$ & 0 \\
\hline $1-3$ months & $78(14.0)$ & $70(12.6)$ & $30(5.4)$ & $10(1.8)$ & 0 & 0 & 0 & $20(3.6)$ & $10(1.8)$ \\
\hline 4-6 months & $100(10.9)$ & $60(6.5)$ & $50(5.4)$ & $10(1.1)$ & 0 & $10(1.1)$ & 0 & $20(2.2)$ & 0 \\
\hline $\begin{array}{l}\text { More than } 6 \\
\text { months }\end{array}$ & $50(15.6)$ & $40(12.5)$ & $10(3.1)$ & 0 & 0 & 0 & 0 & $10(3.1)$ & 0 \\
\hline \multicolumn{10}{|c|}{ Irregular diet time } \\
\hline $\begin{array}{l}4 \text { days per } \\
\text { week }\end{array}$ & $29(21.0)$ & $10(7.2)$ & $10(7.2)$ & 0 & 0 & 0 & 0 & 0 & 0 \\
\hline $\begin{array}{l}2-3 \text { days per } \\
\text { week }\end{array}$ & 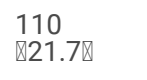 & $\begin{array}{l}70 \\
\square 13.80\end{array}$ & $50 \rrbracket 9.9 \rrbracket$ & $10 \otimes 2.0 \bigotimes$ & 0 & $10 \otimes 2.0 \bigotimes$ & 0 & $20 \rrbracket 3.9 \rrbracket$ & 0 \\
\hline Without & 178ه7.4囚 & 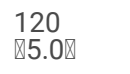 & $50 \rrbracket 2.1 \rrbracket$ & $10 \otimes 0.4 \rrbracket$ & 10冈0.4凹 & 0 & 40囚1.7凹 & $70 \bowtie 2.9 \rrbracket$ & $10 \rrbracket 0.4 \rrbracket$ \\
\hline
\end{tabular}




\begin{tabular}{|c|c|c|c|c|c|c|c|c|c|}
\hline $\begin{array}{l}4 \text { days per } \\
\text { week }\end{array}$ & $60 \rrbracket 33.3 \rrbracket$ & $\begin{array}{l}40 \\
\square 22.2 \rrbracket\end{array}$ & 10囚5.6区 & 0 & 0 & 0 & $10 \rrbracket 5.6 \rrbracket$ & $20 \otimes 11.1 \rrbracket$ & 0 \\
\hline $\begin{array}{l}2-3 \text { days per } \\
\text { week }\end{array}$ & $88 \llbracket 21.1 \rrbracket$ & $40 \otimes 9.6 \rrbracket$ & $30 \otimes 7.2 \rrbracket$ & 0 & 0 & 0 & 0 & $20 \rrbracket 4.8 \rrbracket$ & 0 \\
\hline Without & 169凶6.9凶 & $\begin{array}{l}120 \\
\square 4.9 \rrbracket\end{array}$ & $70 囚 2.8 \rrbracket$ & $20 \rrbracket 0.8 \rrbracket$ & $10 \rrbracket 0.4 \rrbracket$ & 10囚0.4囚 & 30囚1.2囚 & $50 \rrbracket 2.0 \rrbracket$ & $10 \rrbracket 0.4 \rrbracket$ \\
\hline \multicolumn{10}{|c|}{ Time when a serviceman has a chilled abdomen } \\
\hline $\begin{array}{l}4 \text { days per } \\
\text { week }\end{array}$ & $30 \rrbracket 25.0 \rrbracket$ & $\begin{array}{l}30 \\
\square 25.0 \bigotimes\end{array}$ & 0 & 0 & 0 & 0 & $10 \rrbracket 8.3 \rrbracket$ & $10 \rrbracket 8.3 \rrbracket$ & 0 \\
\hline $\begin{array}{l}2-3 \text { days per } \\
\text { week }\end{array}$ & $\begin{array}{l}108 \\
\varangle 34.1 \rrbracket\end{array}$ & $20 \rrbracket 6.3 \rrbracket$ & $50 \otimes 15.8 \rrbracket$ & $20 \rrbracket 6.3 \rrbracket$ & 0 & $10 \rrbracket 3.2 \rrbracket$ & 0 & $30 \bowtie 9.5 \rrbracket$ & $10 \otimes 3.2 \rrbracket$ \\
\hline Without & $179 \llbracket 6.8 \rrbracket$ & 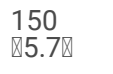 & $60 \bigotimes 2.3 \rrbracket$ & 0 & $10 \rrbracket 0.4 \rrbracket$ & 0 & $30 \rrbracket 1.1 \rrbracket$ & $50 \rrbracket 1.9 \rrbracket$ & 0 \\
\hline \multicolumn{10}{|c|}{ Defiled food history } \\
\hline With & $\begin{array}{l}140 \\
\varangle 21.9 \rrbracket\end{array}$ & 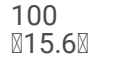 & 70ه11.0囚 & $20 \rrbracket 3.1 \rrbracket$ & 0 & 10ه1.6囚 & 0 & $40 \otimes 6.3 \rrbracket$ & 10ه1.6ه \\
\hline Without & 177ه7.3囚 & $\begin{array}{l}100 \\
\square 4.1 \rrbracket\end{array}$ & 40囚1.7囚 & 0 & $10 \rrbracket 0.4 \rrbracket$ & 0 & 40囚1.7凹 & $50 \rrbracket 2.1 \rrbracket$ & 0 \\
\hline \multicolumn{10}{|c|}{ Prevention knowledge education } \\
\hline With & $189 \otimes 9.4 \rrbracket$ & 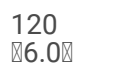 & $50 \otimes 2.5 \rrbracket$ & $10 \otimes 0.5 \rrbracket$ & $10 \otimes 0.5 \rrbracket$ & 0 & $20 \rrbracket 1.0 \rrbracket$ & $60 \rrbracket 3.0 \rrbracket$ & $10 \otimes 0.5 \rrbracket$ \\
\hline Without & 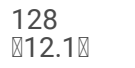 & 80冈7.6ه & $60 \rrbracket 5.7 \rrbracket$ & $10 \rrbracket 0.9 \rrbracket$ & 0 & 10囚0.9囚 & 20ه1.9凶 & $30 \rrbracket 2.8 \rrbracket$ & 0 \\
\hline
\end{tabular}

\section{Logistic regression analysis of risk factors of digestive system diseases of servicemen}

Through regression analysis of statistically significant indicators in univariate analysis, we could know long military service time, officer status, long time of a field training, chilled abdomen, high training pressure and defiled food history had close relations with the occurrence of digestive system diseases.So these factors may be the risk factors of digestive diseases. The details were listed in Table 5.

Table 5

Logistic regression analysis of risk factors of digestive system diseases

\begin{tabular}{|llll|}
\hline Factors & OR & $95 \% \mathrm{Cl}$ & $\mathrm{P}$ \\
\hline Older age & 1.17 & $0.91-1.52$ & 0.23 \\
\hline Shorter military service time & 0.59 & $0.51-0.69$ & 0.00 \\
\hline Officer status & 1.64 & $1.41-1.91$ & 0.00 \\
\hline More cigarette amount & 0.89 & $0.78-1.03$ & 0.11 \\
\hline Family history & 0.86 & $0.62-1.19$ & 0.35 \\
\hline Shorter training time & 0.90 & $0.84-0.98$ & 0.03 \\
\hline Knowledge education & 0.85 & $0.68-1.07$ & 0.16 \\
\hline Irregular diets & 0.78 & $0.62-1.01$ & 0.04 \\
\hline High training pressure & 1.75 & $1.41-2.17$ & 0.00 \\
\hline Chilled abdomen & 1.32 & $1.103-1.69$ & 0.03 \\
\hline Defiled food history & 3.25 & $2.52-4.20$ & 0.00 \\
\hline
\end{tabular}

\section{Discussion}

Looking back at the history of human wars, we can find out that many troops were defeated because of diseases. According to annual statistics from the United States Naval Medical Service, during World War l, gastrointestinal diseases were the third most common diseases that led to American navy servicemen's medical admissions. In World War II, both the American army and navy had a large number of hospital admissions for digestive disorders which were the third most common in the medical diseases ${ }^{[1-3]}$. Digestive diseases were the fourth most common medical disease that caused American navy's hospital admissions during the Korean War.And gastrointestinal diseases were again the third most common diseases that led to American navy servicemen's medical admissions during the Vietnam War.Judging from disease attrition of Chinese army in previous wars,the incidence of the digestive system diseases 
was also very high, which took the first place in the War of Resistance Against Japanese Aggression and in the National War of Liberation, and took the second place in the War of Resistance Against American Aggression and Aid Korea and in Sino-Vitnamese War.In recent years, relevant investigations and studies have shown that the incidence of digestive system diseases of soldiers is still quite high in all medical diseases, of which, functional bowel disease and gastrointestinal ulcer are more common during the training period, and the risk of functional bowel disease may increase with the extension of military service time ${ }^{[4-5]}$. The digestive system is closely related to the nervous system and is sensitive to changes of the external environment. Due to living in a relatively closed and depressing space, and receiving intense physical training,the army servicemen often have great psychological pressure.Therefore, army servicemen are prone to digestive diseases, which can further aggravate their psychological burden, reduce the quality of life, and even seriously affect combat capacity ${ }^{[6]}$. As we know,the study on the spectrum of soldiers' digestive system diseases is quite rare. So the purpose of our study is to investigate and analyze the spectrum of army servicemen's digestive system diseases and find out relevant disease prevention and treatment strategies, so as to protect the health of servicemen and reduce non-combat personnel loss.

This study randomly selected 7 basic units of the army which had a field training in the midland of China and collected data related to the digestive system symptoms and diseases of 3,055 servicemen. It was found that 896 people had symptoms of the digestive system during the training period, with an incidence of $29.3 \%$, and the diarrhea was the most common symptom, with an incidence of $13.7 \%$. A total of 507 servicemen had gastrointestinal diseases, with a prevalence rate of $16.6 \%$, among which, gastritis, enteritis, gastrointestinal ulcers and functional bowel diseases were more common. It is worth mentioning that the incidence of gastroenteritis for servicemen exceeded $15 \%$, which was much higher than that of other diseases, and the result was similar to the finding of previous researchers ${ }^{[6-7]}$. As is known to all, vomiting, poor appetite, diarrhea, abdominal pain,and abdominal distension,were common clinical manifestations of gastroenteritis, which was consistent with our research. Therefore, we have to take measures to decrease the occurrence of gastroenteritis, gastrointestinal ulcer and functional bowel disease during a field training, which include not only hygienic diet and healthy living habits, but also the prevention and treatment of helicobacter pylori $(\mathrm{Hp})$ infection. Hp can destroy the gastric and duodenal mucosal barrier by the self-colonization and secretion of toxins and toxic enzymes. So the standard anti-Hp treatment for patients can effectively reduce the occurrence and recurrence of gastritis and gastrointestinal ulcer ${ }^{[8]}$. During the field training, the army servicemen have intense physical training in the tough environment, because the regions in the midland such as Hubei and Shaanxi Province are rainy, humid and very hot. And long-term high temperature and high pressure working environment increase the risk for functional bowel disease. Moreover, the army servicemen often have inadequate sleep time, which also increase the occurrence of functional bowel disease ${ }^{[9]}$. Hence, for servicemen who always have training tasks every year, it's quite necessary to receive regular blood test and abdominal ultrasound examination. To detect abnormal indicators early, and then adjust the diet structure timely, have a appropriate rest, and accept early intervention and treatment can prevent the progress of liver and gallbladder diseases.

In this study, we focused on the comparison of the digestive system symptoms and diseases for military officers and soldiers in different groups, and then found out there were significant differences in the incidence of digestive symptoms and diseases of servicemen among different groups of age, military service time, status, smoking amount,digestive disease family history,the field training time, irregular diet times,training pressure,chilled abdomen,defiled food history and disease prevention knowledge education( $\mathrm{P} \otimes 0.01)$.but through regression analysis of statistically significant indicators in univariate analysis, we could know long military service time, officer status, long time of a field training, chilled abdomen, high training pressure and defiled food history had close relations with the occurrence of digestive system diseases.So these factors may be the risk factors of digestive diseases. In the army, veterans and military officers need to carry out more important tasks and more responsibility, that may explain the long service time and identity of military officer are closely related with the occurrence of digestive diseases. And some researchers also discover long time of service is the risk factor for functional bowel disease ${ }^{[10-11]}$. A study of the US Army also confirms that the prevalence of functional bowel disorder for soldiers is related to stressors, the service years is one of which ${ }^{[12]}$. However, the investigation results in the basic units of the air force shows that the risk of functional bowel disease peaks at the age of 18-25 and over 41 , which is quite different from the onset age of army as we discuss before ${ }^{[13-14]}$. This seems to suggest that there are differences in the characteristics of the disease among different services, which still needs further verification. The lectures on prevention and treatment knowledge can strengthen awareness of servicemen for digestive diseases, understand the characteristics, inducements and simple diagnosis and treatment rules of diseases. Our study also confirmed this view, the publicity of prevention and treatment knowledge once or twice a year can reduce the incidence of digestive diseases.

Non-steroidal drugs inhibit the synthesis of prostaglandin by inhibiting coxidase, which leads to the disruption of gastrointestinal mucosal balance and causes mucosal inflammatory lesions. Therefore, the long-term use of non-steroidal drugs is a risk factor for the occurrence of digestive system diseases. But in our study, there was no significant difference in the incidence of digestive symptoms and diseases between different groups of NSAIDs use history(Pख0.05) Maybe it is associated with shorter duration of non-steroidal drug use, and after all, the time of a field training is generally not more than half a year.Also it may be related with the sample size, because the number of military personnel taking non-steroidal drugs is relatively less. Some studies believe that there is no clear relationship between smoking and the occurrence of digestive system diseases such as functional bowel disease ${ }^{[14-15]}$. The study also found that smoking did not increase the risk of digestive diseases by regression analysis. However, other studies have confirmed that large smoking amount is a risk factor for digestive system diseases ${ }^{[11015]}$. So the relation remains to be confirmed by large-scale epidemiological studies.

In addition to focusing on the possible risk factors discussed in this study, the training on skills for military doctors such as online teaching and technical guidance from medical experts, is also very important for the prevention and treatment of digestive system diseases. Expanding the number coverage of gastroscopy, accelerating the development of $5 \mathrm{G}$ network application in endoscopy and the promotion of portable endoscopy will also help improve the professional level of medical personnel in the health center and the diagnosis and treatment rate of diseases.

As a regression investigation, this study cannot clearly determine the causal relationship between digestive system symptoms and diseases. Besides, digestive system symptoms and diseases in the questionnaire are mainly from servicemen's clinic experience,not from doctors' real-time diagnoses, which may be not reliable,and then lead to the biased investigation. So the next step is to follow up servicemen and conduct a large-scale prospective investigation and study. 


\section{Conclusions}

During a field training, attention should be paid to the military personnel with long military service time or a family history of digestive diseases and officers. A healthy and regular diet, the thermal outfit,appropriate decompression of negative emotions and reasonable training time may effectively decrease the risk of digestive diseases, so that to reduce attrition.

\section{Declarations}

\section{Ethics approval and consent to participate}

All the experiment protocol for involving human data was in accordance to Declaration of Helsinki.

All experimental protocols for using human data (demographic data) were approved by Ethics Committee of PLA General Hospital.

Informed consent was obtained from all adult patients and legally authorized representatives of minors less than age 16 years.

\section{Consent for publication}

All authors have read and approved the final version to be published.

\section{Availability of data and materials}

The data used and analysed in the study available from the corresponding author on the reasonable request.

\section{Competing interests}

All authors declare that they have no competing interests.

\section{Authors' contributions}

Our research was mainly designed by MY Li and GJ Zhang; F Lin, YZ Zhang, HH Li,

HW Song and B Li distributed questionnaires, and collected data; GJ Zhang analyzed the data and wrote the manuscript; MY Li provided analytical oversight and revised the manuscript for important intellectual content.

\section{Funding}

Independent research project of field internal medicine in the construction of key military disciplines during the 13th Five-Year Plan.

\section{Acknowledgements}

The authors sincerely thank all staff members of the army units selected by us, for their help in our study.

\section{References}

1. Li ZS,Mei CL囚Modern Field Internal Medicine[M].Shanghai囚Shanghai Science and Technology Press.2013囚2-6区

2. Liu GD, Wang N, Wang HM, Li X, Shao JJ, Liu ZF, Jiang M, Wang L, Wang ZK, Li M, Cao XY, Wang J, Zhang R, Chen YD. Military medical research on internal diseases in modern warfare: new concepts, demands, challenges, and opportunities. Mil Med Res. 2021 Mar 12;8(1):20.

3. Howard JT, Kotwal RS, Stern CA, Janak JC, Mazuchowski EL, Butler FK, Stockinger ZT, Holcomb BR, Bono RC, Smith DJ. Use of Combat Casualty Care Data to Assess the US Military Trauma System During the Afghanistan and Iraq Conflicts, 2001-2017. JAMA Surg. 2019 Jul 1;154(7):600-608.

4. Yan B,Cui LH,Peng LH,Guo X,Sun G,Wang WF,Yang YS.An epidemiological research and risk factor analysis of functional gastroduodenal disorders in a Chinese naval force[J]. Mil Med Res.2013,38(06):446-449.

5. Koloski NA, Jones M, Walker MM, Holtmann G, Talley NJ. Functional dyspepsia is associated with lower exercise levels: A population-based study. United European Gastroenterol J. 2020 Jun;8(5):577-583.

6. Xiong LS, Shi Q, Gong XR, Cui Y, Chen MH. The spectra, symptom profiles and overlap of Rome III functional gastrointestinal disorders in a tertiary center in South China. J Dig Dis. 2014 Oct;15(10):538-44.

7. Bang CS, Kim YS, Han JH, Lee YS, Baik GH, Kim JB, Suk KT, Yoon JH, Kim DJ. Functional Gastrointestinal Disorders in Young Military Men. Gut Liver. 2015 Jul;9(4):509-15 
8. Camilo V, Sugiyama T, Touati E. Pathogenesis of Helicobacter pylori infection. Helicobacter. 2017 Sep;22 Suppl 1. Abadi AT, Kusters JG. Management of Helicobacter pylori infections. BMC Gastroenterol. 2016 Aug 12;16(1):94.

9. Li MY,Wang ZQ®Sun G,Yang YS.Morbidity analysis of functional gastrointestinal disease in soldiers in the area affected by the Wenchuan earthquake[J].World Chinese Journal of Digestion. 2010,18(15):1594-1597.

10. Mahmood K, Riaz R, Ul Haq MS, Hamid K, Jawed H. Association of cigarette smoking with irritable bowel syndrome: A cross-sectional study. Med J Islam Repub Iran. 2020 Jul 1;34:72.

11. Yao X,Jin HF,Zhi J,Wang N,Liu Z,Sun R.Clinical features of unspecified functional bowel disorder in servicemen from a Chinese army unit[J].Journal of PLA Medical College.2019,40(05):401-406.

12. Riddle MS, Welsh M, Porter CK, Nieh C, Boyko EJ, Gackstetter G, Hooper TI. The Epidemiology of Irritable Bowel Syndrome in the US Military: Findings from the Millennium Cohort Study. Am J Gastroenterol. 2016 Jan;111(1):93-104.

13. Papaefthymiou A, Doulberis M, Kountouras J, Kolokytha C, Galanopoulos M, Liatsos C, Kyriakos N, Giakoumis M, Papadomichelakis M, Polyzos SA, Kotronis G, Katsinelos P. Impact of occupational stress on irritable bowel syndrome pathophysiology and potential management in active duty noncombat Greek military personnel: a multicenter prospective survey. Eur J Gastroenterol Hepatol. 2019 Aug;31(8):954-963.

14. Le Pluart D, Sabaté JM, Bouchoucha M, Hercberg S, Benamouzig R, Julia C. Functional gastrointestinal disorders in 35,447 adults and their association with body mass index. Aliment Pharmacol Ther. 2015 Apr;41(8):758-67.

15. Ohlsson B. The role of smoking and alcohol behaviour in management of functional gastrointestinal disorders. Best Pract Res Clin Gastroenterol. 2017 Oct;31(5):545-552. 